\title{
TRATAMENTO DE CISTO MESENTÉRICO QUILOSO
}

\author{
Mesenteric chylous cyst treatment
}

Leandro Tibúrcio REGINA, Gustavo Mariani BOLINA, Luiz Ronaldo ALBERTI, Andy PETROIANU

Trabalho realizado no Serviço de Cirurgia Geral da Santa Casa de Belo Horizonte, Belo Horizonte, MG, Brasil
RESUMO - Introdução - Cistos mesentéricos são tumores abdominais raros, que podem acometer pacientes de todas as faixas etárias, sendo mais comuns em mulheres na quarta década de vida. Essas neoplasias são geralmente assintomáticas e diagnosticadas por acaso. Seu tratamento é baseado na retirada do cisto, por laparotomia ou laparoscopia, embora em alguns casos possa ser necessária a ressecção de segmento intestinal. $O$ prognóstico é bom e a recorrência é baixa. Relato do caso - Mulher de 40 anos de idade, obesa, hipertensa e diabética, apresentou história de dor abdominal contínua de pouca intensidade no hipocôndrio esquerdo e epigástrio, sem irradiações, durante cerca de três meses. Após um mês do início dos sintomas percebeu massa em andar superior do abdome palpável desde o epigástrio até o flanco esquerdo, de consistência endurecida, pouco móvel, superfície regular e dolorosa. A tomografia computadorizada de abdome evidenciou formação cística de paredes finas e lisas, medindo $12,9 \times 11,6 \times 9,9 \mathrm{~cm}$, situada em flanco esquerdo, em região mesentérica, deslocando estruturas adjacentes, sugestiva de cisto mesentérico. À laparotomia evidenciou-se massa cística, com aproximadamente $10 \mathrm{~cm}$ de diâmetro, de parede fina e lisa, cor amarelada, com vasos tortuosos na superfície, sem aderência à estruturas adjacentes, localizada no mesojejuno a cerca de $50 \mathrm{~cm}$ da flexura duodenojejunal. O cisto foi completamente retirado e seu conteúdo era líquido brancacento e inodor, sugestivo de quilo. O exame anatomopatológico revelou cisto mesotelial benigno mesentérico. Conclusão - O tratamento desses cistos consiste basicamente na sua retirada ou descapsulação, que pode ser realizado de preferência por via laparoscópica.
DESCRITORES - Cisto mesentérico Cirurgia. Tratamento.

\section{Correspondência: \\ Andy Petroianu \\ e-mail: petroian@medicina.ufmg.br \\ Fonte de financiamento: não há \\ Conflito de interesses: não há}

Recebido para publicação: 25/08/2009

Aceito para publicação: 15/12/2009

HEADINGS - Mesenteric Cyst. Surgery. Therapeutics.
ABSTRACT - Introduction - Mesenteric cysts are rare abdominal tumors that may occur in patients at any age and are more common in women at the age of forty. They are generally free of symptoms and incidentally found. The treatment is based on cyst ressection, by laparotomy or laparoscopy, although the enterectomy may be necessary. The prognosis is usually good and the recurrence is rare. Case report - Women 40 years of age, obese, hypertensive and diabetic, had a history of continuous abdominal pain of low intensity in the left hypochondrium and epigastrium, without irradiation, for about three months. After a month of onset of symptoms noticed a mass in the upper abdomen palpable from the epigastrium to the left flank with a hard consistency, little mobility, smooth surface and painful. Computed tomography of the abdomen showed cystic formation with thin smooth wall, measuring $12.9 \times 11.6 \times 9.9 \mathrm{~cm}$, located on the left flank in the mesenteric region, displacing adjacent structures, suggestive of mesenteric cyst. At laparotomy a cystic mass was found, with approximately $10 \mathrm{~cm}$ in diameter, yellowish, with tortuous vessels on the surface, without adherence to adjacent structures, located in mesojejunum about $50 \mathrm{~cm}$ from the duodenojejunal flexure. The cyst was completely removed and its content was whitish and odorless liquid, suggesting a chylo. Pathology revealed benign mesothelial mesenteric cyst. Conclusion - The treatment of these cysts consists basically of their withdrawal or decapsulization, which can preferably be achieved by laparoscopy.
$\mathrm{O}$ s cistos de mesentério são tumores abdominais raros, que podem acometer indivíduos de qualquer faixa etária. Há predomínio do sexo feminino, na quarta década de vida e geralmente não causam sintomas, sendo diagnosticados ocasionalmente. São classificados em linfangiomas ou cistos mesoteliais e podem originar-se de tecidos linfáticos, vascular, nervoso e conjuntivo. O tratamento consiste em sua retirada ou abertura ampla. Em alguns casos, pode ser necessária enterectomia segmentar. O prognóstico é bom. $\mathrm{O}$ objetivo deste trabalho é apresentar o caso de paciente portadora de um cisto quiloso volumoso, no mesojejuno, ressecado através de laparotomia. 


\section{RELATO DO CASO}

Mulher de 40 anos de idade, obesa, hipertensa e diabética, deu entrada no Serviço de Cirurgia Geral da Santa Casa de Belo Horizonte com história de dor abdominal contínua e de pouca intensidade, no hipocôndrio esquerdo e epigástrio, sem irradiações, durante cerca de três meses. A dor melhorava com analgésicos comuns, associava-se a episódios de náuseas, vômitos e plenitude pós-prandial. Após um mês do início dos sintomas ela percebeu massa em andar superior do abdome. Não houve trauma na região da massa, febre, melena ou hematoquezia.

A massa era palpável desde o epigástrio até o flanco esquerdo, de consistência endurecida, pouco móvel, superfície regular e dolorosa. A tomografia computadorizada de abdome evidenciou formação cística de paredes finas e lisas, medindo $12,9 \times 11,6 \times 9,9$ $\mathrm{cm}$, situada em flanco esquerdo, em região mesentérica, deslocando estruturas adjacentes, sugestiva de cisto mesentérico (Figura 1A). À laparotomia evidenciouse massa cística, com aproximadamente $10 \mathrm{~cm}$ de diâmetro, de parede fina e lisa, cor amarelada, com vasos tortuosos na superfície, sem aderência à estruturas adjacentes, localizada no mesojejuno a cerca de $50 \mathrm{~cm}$ da flexura duodenojejunal (Figura 1B). O cisto foi completamente retirado e seu conteúdo era líquido brancacento e inodor, sugestivo de quilo. O exame anatomopatológico revelou cisto mesotelial benigno mesentérico (Figura1C).

A paciente evoluiu satisfatoriamente, recebendo alta no segundo dia pós-operatório, assintomática.

\section{DISCUSSÃO}

Os cistos mesentéricos são tumores abdominais raros. Há cerca de 820 casos publicados na literatura desde sua primeira descrição em 1507, por Benevieni, anatomista italiano, em necropsia de uma menina de oito anos de idade ${ }^{3,6,7,9,10}$. A primeira descrição de cisto mesentérico quiloso data de 1842, por Rokitanski². Tillaux, em 1880, realizou a primeira ressecção com sucesso de um cisto mesentérico e em 1883 Péan realizou a primeira descapsulação ${ }^{7,11}$.

A incidência de tratamento desses cistos é de um caso para cada 100.000 a 250.000 internações hospitalares $5,6,6,10,11$. Eles se manifestam em qualquer faixa etária, porém, dois terços são diagnosticados em maiores de 10 anos de idade, com predominância do sexo feminino, na terceira e quarta décadas de vida $a^{6,7,11}$.

Os cistos podem ocorrer em qualquer parte do intestino desde o duodeno até o reto, contudo a maior parte acomete o íleo seguido pelo cólon ascendente, sendo os cistos quilosos mais frequentes no intestino delgado e os serosos no cólon $n^{1,6,7,10}$. Sua etiologia ainda não está esclarecida, mas acredita-se que possam derivar de malformações de ductos linfáticos
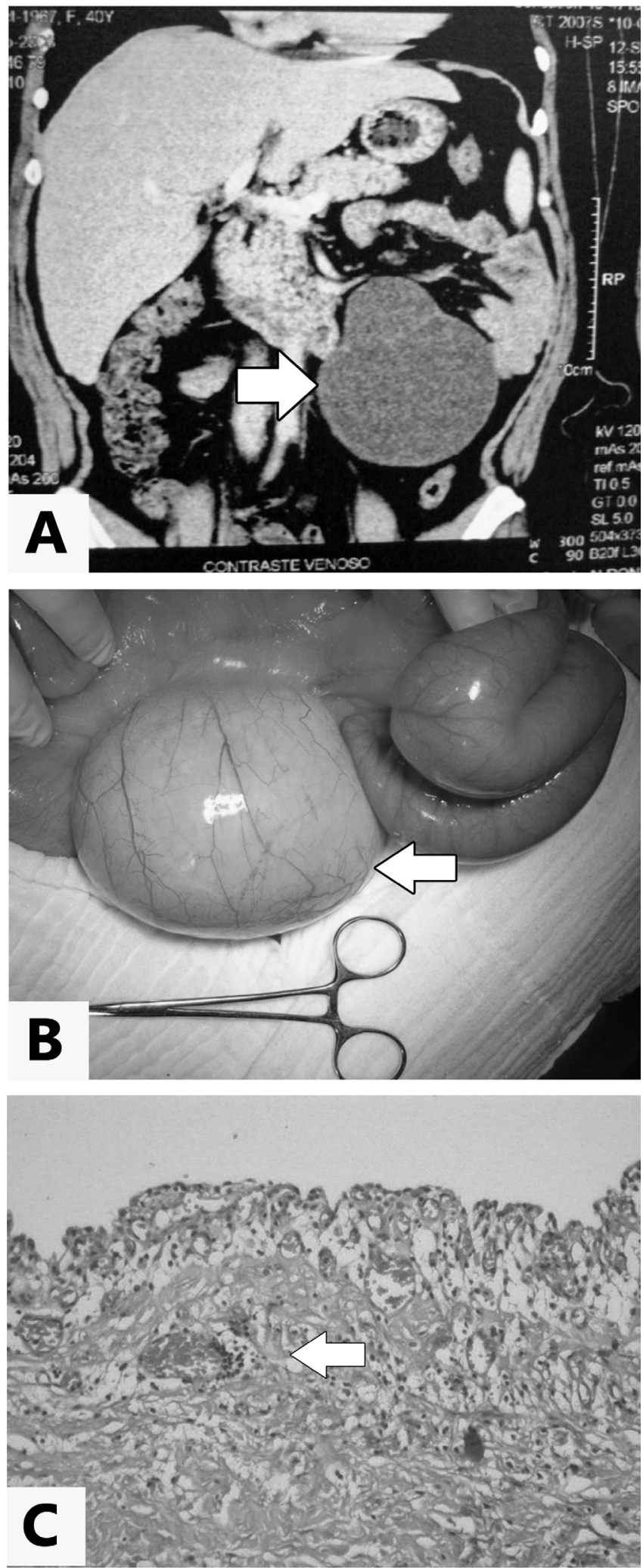

FIGURA 1 - A -Tomografia computadorizada de abdome evidenciando lesão cística em flanco esquerdo do abdome deslocando alça intestinal adjacente. B - Imagem operatória da massa cística de mesojejuno. C - Imagem microscópica (HE) da parede do cisto mostrando tecido fibroconjuntivo com áreas de hemorragia, fibrose e infiltrado inflamatório. 
abdominais em fundo cego ou serem sequelas de trauma, infecções ou neoplasias ${ }^{5,10}$.

A maior parte dos casos é assintomática sendo descoberta incidentalmente durante propedêutica para outras afecções, todavia podem apresentarse com quadro de dor abdominal aguda ou crônica, obstrução intestinal, náuseas e vômitos ou até mesmo como quadro de abdome agudo e choque devido à ruptura, infecção ou torção $0^{5,6,9,10}$. O diagnóstico é suspeitado no exame do abdome, com o auxílio do sinal de Tillaux, mobilização do cisto como um todo à palpação abdominal profunda 4 . A confirmação ocorre com ultrassonografia e tomografia computadorizada que auxiliam na diferenciação entre massas císticas e sólidas e na avaliação de comprometimento de estruturas adjacentes ${ }^{5,8,10,11,13}$.

Testes laboratoriais não são úteis nesses casos. O diagnóstico diferencial é feito com linfangiomas e mesoteliomas. Sua degeneração malígna é rara, 3 a 4\% dos casos ${ }^{9}$.

O tratamento consiste basicamente na retirada do cisto ou sua descapsulação, acompanhada de biópsia da cápsula, que pode ser realizado de preferência por via laparoscópica ${ }^{2,12}$. A recorrência chega a 13,6\%, estando relacionada com a técnica operatória. O seguimento pós-operatório é realizado por meio de exames ultrassonográficos periódicos ${ }^{5,9}$. O prognóstico é bom, com remissão total dos sintomas ${ }^{10}$.

\section{CONCLUSÃO}

O tratamento desses cistos consiste basicamente na sua retirada ou descapsulação, que pode ser realizado de preferência por via laparoscópica

\section{REFERÊNCIAS}

1. Adams JT. Parede Abdominal, Omento, Mesentério e Retroperitônio. In Scwarts SI. Princípios de Cirurgia. $5^{a}$ Ed, Rio de Janeiro: Guanabara Koogan;1991. p.1264-1266.

2. Al-Haifi MB, Abdulsmad AM, Juma TH. Laparoscapic Excision of Mesenteric Cyst: Case Report. Kuwait Medical Journal 2007;39(2):167-169.

3. Block FB. Chylous mesenteric cyst: Case report. Annals of Surgery 1948; 128:158-160.

4. Cônsolo FS, Cardoso AAP, Monge M, Santos P, Hernandez PM. Formação Cística de Túnica Serosa de Cólon Ascendente - Relato de caso. RAMB 2005;55(1/2),36-39.

5. Duldulao MP, Thiruchitrambalam A, Kaul A. Mesenteric cyst: A rare cause of lower abdominal pain. Surg Rounds 2008; 31(3): 123-127.

6. Filho FAC, Landim FM, Perdigão FB. Linfangioma Cístico do Mesentério: Uma Rara Apresentação de Abdômen Agudo. Rev. Col. Bras. Cir. 1999;27(2):139-140.

7. Fraga MR, Cavalcante AFS, Benício IB, Pereira AA, Leite CAC, Dias RP. Cisto Mesentérico. Rev. Ped. Ceará 2004;5(1):34-37.

8. Fujita N, Noda Y, Kobayashi G. Chylous cyst of the mesentery: US and CT diagnosis. Abdom. Imaging 1995;20(3):259-261.

9. Garcia RP, López ET, Ovalle JLR. Quiste mesentérico: Descripción de um caso. Cirurjano General 2001; 23(2): 109-112.

10. Miliaras S, Trygonis S, Papandoniou A, Trygonis C, Kiskinis D. Mesenteric Cyst of the Descending Colon: Report of a Case. Acta chir belg 2006;106: 714-716.

11. Miljikovic D, Gmijovic D, Radojkovic G, Radovanovic Z. Mesenteric cyst. Arch Oncol 2007; 15(3-4): 91-93.

12. Sahin DA, Akbulut G, Saykol V, San O, Tokyol C, Dilek ON. Laparoscopic Enucleation of Mesenteric Cyst: A Case Report. The Mount Sinai Journal of Medicine 2006;73(7);1019-1020.

13. Simeone DM, Pranikoff T, Mesenteric Cyst. Images in Clinical Medicine 1999;341(9):659. 